Proceedings of Robotics 98, the 3rd Conference and Exposition on Robotics in Challenging Environments, Albuquerque, New Mexico, April 26-30, 1998

\title{
Design and Implementation of an Innovative Micro-Rover
}

\author{
Roland Siegwart ${ }^{1}$, Michel Lauria ${ }^{1}$, Pierre-Alain Mäusli $^{2}$, Michel Van Winnendael $^{3}$
}

\begin{abstract}
Mobile robots are of high interest for unmaned planetary exploration. The very successful Pathfinder mission to Mars has impressively demonstrated the potential of mobile platforms for planetary exploration [1]. The European Space Agency (ESA) also started to develop concepts for micro-rovers for Mars missions. Within an interdisciplinary group of companies specialized in space applications and research labs new designs of micro-rovers have been investigated. Two concepts, a simple and robust one and an innovative one, have been selected and functional breadboard models of them are currently built.

After a discussion of the key issues for robust locomotion the present paper will focus on the design and control of the more innovative solution. It consists of 6 independently driven wheels arranged in two triangles. It therefore allows not only for efficient rolling on flat surfaces but also to step on obstacles. Additionally the center of mass and the instrumentation carrousel is adjustable, allowing to optimally balance the micro-rover in almost every situation. Even after flipping over the robot will always be able to get back on its wheels.
\end{abstract}

\section{INTRODUCTION}

For the future planetary missions to Mars and Moon the European Space Agency (ESA) is seeking for micro-rovers to explore an area with a radius of the order of $10 \mathrm{~m}$ around the lander [2]. Detailed background information can be found in [3], [4]. This micro-rover should be able to move to objects, to make measurements with its on board instruments and to transmit the information back to the lander. To come up with new innovative solutions a team of companies and research labs was selected. The goal of the project is to develop new concepts, to select the two most promising ones (a simple and robust one and a more innovative one) and to build prototypes for verification of their performances.

\subsection{Requirements}

Within the framework of the current development ESA defined the following main requirements and constraints:

- Stowed dimensions $(\mathrm{cm})$ :

- Net mass of the robot system:

- Mass of the scientific payload:

- Electrical power provided by lander:

- Maximum obstacle height to overcome:

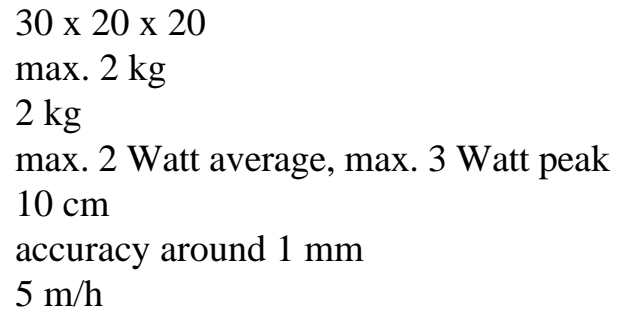

- Possibility to positio

The rover must overcome obstacles of $0.1 \mathrm{~m}$ height and holes of $0.1 \mathrm{~m}$ width. It has to be able

1.Swiss Federal Institute of Technology Lausanne (EPFL), Switzerland

2. MECANEX S.A., Nyon, Switzerland

3.ESA/ESTEC, Noordwijk, The Netherlands 
to climb up slopes of $15^{\circ}$ and down $20^{\circ}$. Simple maneuvers like turning and moving backward is required too. These specifications related to the terrain can be used as a benchmark. To ensure functionality in real martian terrain, extended tests in various environments will be made. To ensure excellent performance the following important issues have been identified and specified:

- No risk of sinkage and getting stuck in the sand

- Flip-over stability, stability against wind

- Recovery after flip-over and after having been buried by a sand storm

\section{DISCUSSION OF THE LOCOMOTION CONCEPTS}

In various research projects all over the world the different locomotion concepts for mobile robots have been analyzed and new concepts have been proposed [5][6]. Good general surveys are provided in [5][7][8][9][10][11][12]. In [8] over 300 mobility concepts and more than 400 navigation concepts have been generated and discussed, yielding to three candidate systems, a symmetrical walker with six identical legs, a four-wheeled vehicle and a so called attached scout concept with six wheels.

Four main principles - rolling, walking, crawling and jumping - have been identified for full or partial solid state contact. However, additional locomotion principles without solid state contact could be of interest in special environment.

Most of the mobile robot for planetary exploration will move most of their time on nearly flat surfaces, where rolling motion has its highest efficiency and performance. However, some primitive climbing abilities are required in many cases. Therefore hybrid approaches, where for example rolling motion is combined with stepping, are of high interest.

Table 1 gives an overview of characteristics of the different locomotion concepts. The scoring represents our personal opinion and is of course not unbiased. As can be seen, the rolling locomotion has only little disadvantages, mainly concerning the surmounting of objects. With the proposed hybrid approach this weak point is solved, but the robustness is lowered. The 'Stepping Triple Wheels' which is also considered in the table was selected as the most promising candidate for the innovative solution.

Table 1: Judgement of the different locomotion concepts

\begin{tabular}{|c|c|c|c|c|c|c|c|}
\hline $\begin{array}{c}\text { Specification } \\
\text { Concept }\end{array}$ & $\begin{array}{c}\text { Minimum } \\
\text { No. of } \\
\text { motors }\end{array}$ & Volume & $\begin{array}{c}\text { Energy } \\
\text { consump- } \\
\text { tion }\end{array}$ & $\begin{array}{l}\text { Turnover } \\
\text { stability, } \\
\text { recovery }\end{array}$ & $\begin{array}{l}\text { Surmount- } \\
\text { ing of } \\
\text { objects }\end{array}$ & $\begin{array}{c}\text { Robust- } \\
\text { ness }\end{array}$ & $\begin{array}{l}\text { Inherent } \\
\text { complex- } \\
\text { ity }\end{array}$ \\
\hline $\begin{array}{l}\text { Rolling - wheels } \\
\text { - caterpillars }\end{array}$ & $\begin{array}{l}2-3 \\
2-3\end{array}$ & $\begin{array}{l}\mathrm{O} \\
-\end{array}$ & $\begin{array}{l}+ \\
-\end{array}$ & $\begin{array}{l}\mathrm{O} \\
\mathrm{o}\end{array}$ & $\begin{array}{l}- \\
\text { o }\end{array}$ & $\begin{array}{l}+ \\
+\end{array}$ & $\begin{array}{l}+ \\
0\end{array}$ \\
\hline Walking & $>3$ & + & - & o & + & - & -- \\
\hline Crawling & 3 & + & -- & $\mathrm{O}$ & - & o & o \\
\hline Jumping & 3 & o & - & -- & ++ & - & - \\
\hline Trailing & (1) & ++ & + & -- & + & -- & + \\
\hline \multicolumn{8}{|c|}{ Proposed hybrid solution: 'Stepping Triple Wheels' } \\
\hline Triple Wheels & 4 & o & + & + & + & o & - \\
\hline
\end{tabular}

\section{THE STEPPING TRIPLE WHEELS}

\subsection{Mechanical Design}

Our locomotion concept is a hybrid approach called stepping triple wheels (figure 1) because of both, walking and rolling capabilities similar to AIMARS [13]. Two independently driven sets of three wheels are supported by two frames. The frames can rotate independently around the main body (payload frame) and allow the rover to actively lift one wheel to step on objects. 

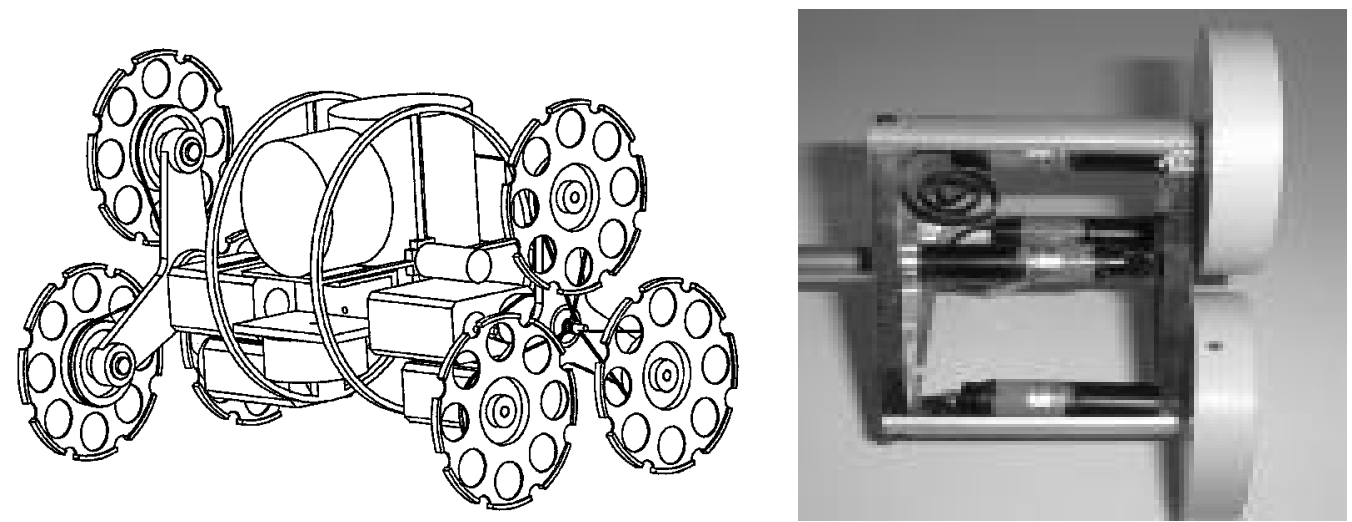

FIGURE 1. Stepping triple wheels concept (left)

The three wheels and the four motors of one frame of the rover (right)

The mechanical design of the rover can be separated in two different parts, the wheel frame and the payload frame, which can be designed independently. The first part regards the wheels and the frame which support them. The second part concerns the scientific payload and its support. Concerning the wheel frames a minimum of four degrees of freedom (DOF) are required. Two of them are need to allow the vehicle to move in the plane. To provide a primitive walking capability two additional degrees of freedom are added. There are a lot of possibilities to implement such a mechanism. In the presented design 8 motors are used so that each wheel and frame can be driven independently. The advantages are high redundancy and a simpler design. Figure 1 right show the three wheels and the four motors of the wheel frame.

The payload frame of the rover contains all the scientific instruments necessary to accomplish the mission. With $2 \mathrm{~kg}$ the payload is the most important part of the total rover mass. By moving the payload frame relatively to the wheel frames the center of gravity of the rover can be adjusted (see figure 3 ). This adds new capabilities for locomotion, e.g. to climb on obstacles or to recover after flipping over (see figures 3 to 5 ).

To make optimal measurements it is required to position all the different instruments on exactly the same point of scientific interest. With the proposed micro-rover all the instruments are mounted in a carrousel in which they are headed radially (figure 1 left). The change from one instrument to an other is achieved by only rotating the payload frame around its symmetry axis. It is realized by introducing an additional motor within the centre of the payload frame. Thus, the instrument change can be made maintaining high accuracy, without any displacement of the vehicle.

\subsection{Electronic design}

To control the different motors and sensors an appropriate electronic must be integrated in the rover. Rotating contacts (sliprings) are used to connect the individual circuits on the frames. Each rotating contact has two leads for power supply and two for a serial interface. The detailed architecture of the electronic sub-system is described in the scheme of figure 2 .

The electronics of the robot system is composed of four separated modules which communicates each other via a serial link. Three modules are in charge of the motors and sensors control. The role of the last module is to coordinate the others, to take higher level decisions and to communicate with the lander.

\subsection{Locomotion}

As mentioned before, the stepping triple wheels concept is an hybrid approach, which allows for efficient rolling and to stepping over objects. In the following figures different maneuvers are presented.

Figure 3 (right) explains the different stages during stepping on an object. To get on the object 
the center of gravity of the rover is moved outside the contact surface formed by the four wheels. Thus the rover gets out of balance and 'falls' with its upper wheel onto the object.

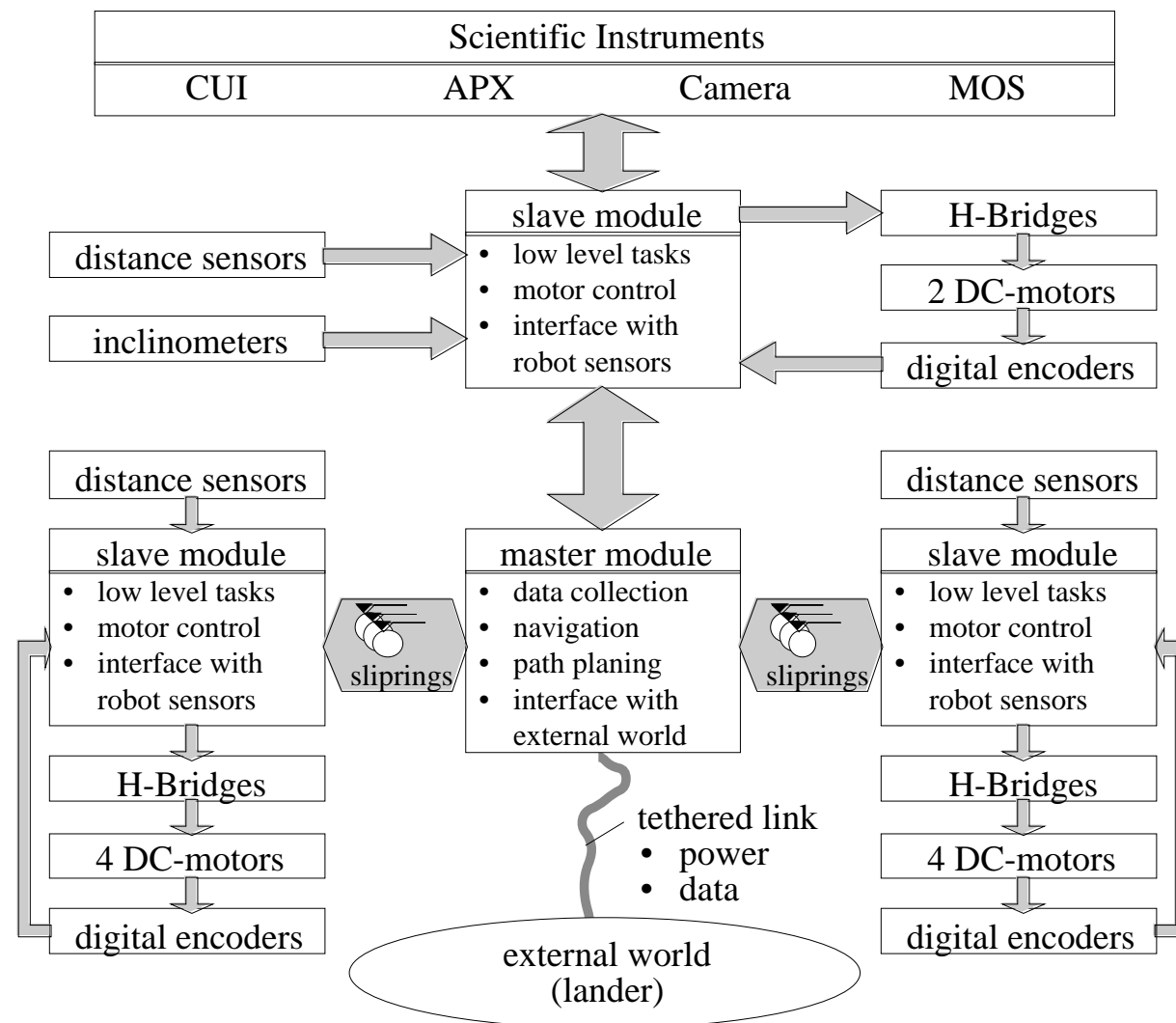

FIGURE 2. Electronics scheme of the micro-rover
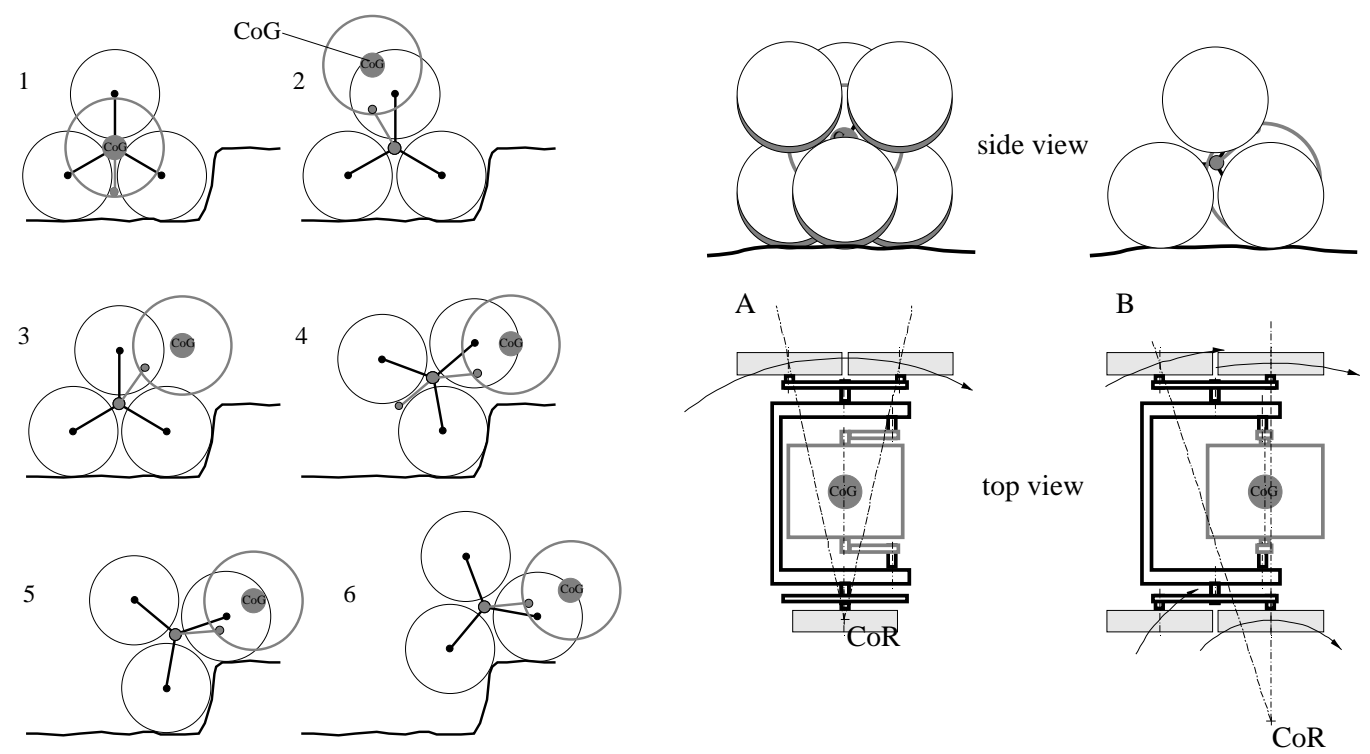

FIGURE 3. Left: Locomotion concept: Stepping on an object For simplicity the Center of Gravity (CoG) is assumed to be equal to that of the payload

Right: Locomotion concept: Turning (CoR: Center of Rotation)

A: turning on three wheels

$B$ : turning on four wheels with main load on front wheels

4 Siegwart, Lauria, Mäusli, Van Winnendael 
As can be seen in figure 4, no displacement of the center of gravity is required if the rover moves over a small rock $(<10 \mathrm{~cm})$. Therefore, small object can be passed without any special control commands.

Turning can be done by applying two different speeds to the wheels. Friction on the wheels is considerably reduced either by rotating the two wheel-frames so that only one wheel is in ground contact at the point of rotation (figure 3 right A) or by adjusting the centre of gravity to the appropriate place close to the front wheels (figure 3 right $\mathrm{B}$ ). With the concept of figure 3 right $\mathrm{B}$ all 4 wheels are maintained on the ground, but the payload is displaced so that the centre of gravity is essentially on the two front wheels. The different rotation velocities on the two front wheels configure the trajectory and the residual low forces on the two others wheels will reduce the friction on ground to a very low level.

An other very important aspect concerns the ability to recover after flipping over. By moving the main mass out, the centre of gravity can be placed outside the base formed by the three wheels. This allows to bring the rover back on its wheels (figure 5).
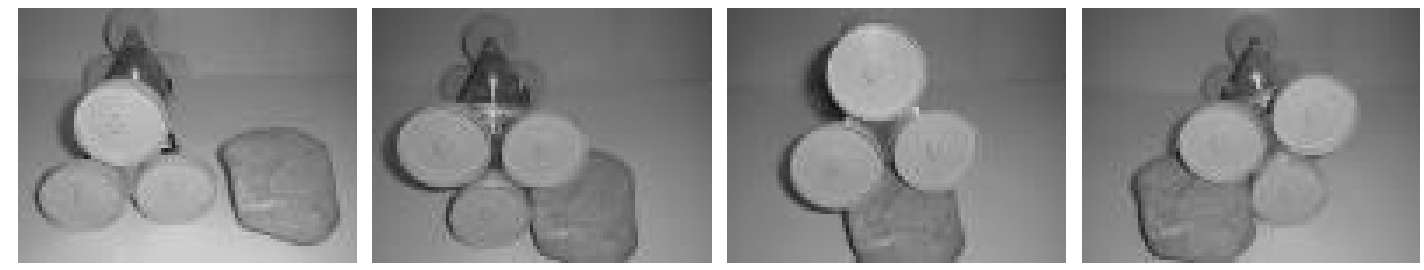

FIGURE 4. Real movements of the robot while stepping over an object

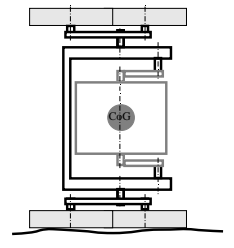

1
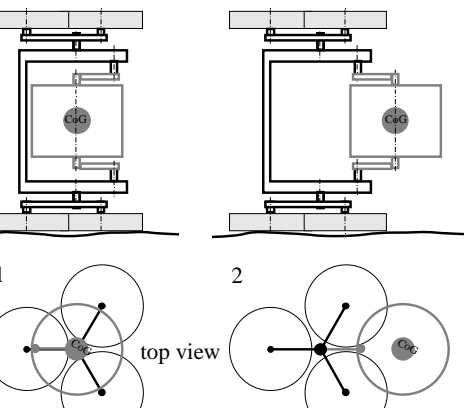

2

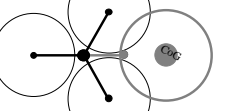

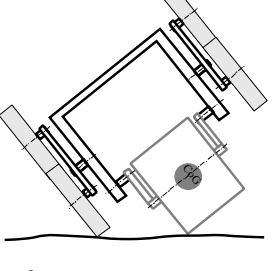

3

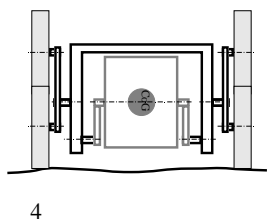

FIGURE 5. Recovery after flipping over:

1: initial position with CoG within central axis

2: movement of the CoG to its most outside position -> rover flips back on wheels

3: situation just after flipping back on wheels

4: situation after moving the CoG back to its initial position

\section{CONTROL OF THE MICRO-ROVER}

Control is assumed to be distributed over the rover, the lander and ground (Earth). Various architectures and concepts, some of them more hierarchical [14], [15] and some of them more behavior based [16], [17], have been proposed for space robots. Our approach fits within the unified control architecture for planetary rovers which has been proposed in [18].

The degree of autonomy of a robot depends directly of the complexity of its local control. Totally autonomous mobile systems are very hard to build because of energy and navigation problems. In our case, a semi-autonomous robot is more adapted to fulfill the mission's requirements.

A crucial aspect of the whole system is the low transmission rate from Mars to Earth. A teleoperator should act only for path planning and in case of hazards. Regularly, all decisions are made by the lander and/or the rovers. Local intelligence must therefore exceed the reflexive behaviors and provide problem solving for more complex situations. An essential skill of the rov- 
er is the ability to decide when and how to avoid or surmount an obstacle. The application of complex sensors like stereo vision, structured light or 3D laser scanners is doubtful considering the specifications of mass, volume, energy consumption or reliability. We propose the implementation of simple, ant-like intelligence on the rover and a global position update based on the vision signals acquired on the lander (figure 6).

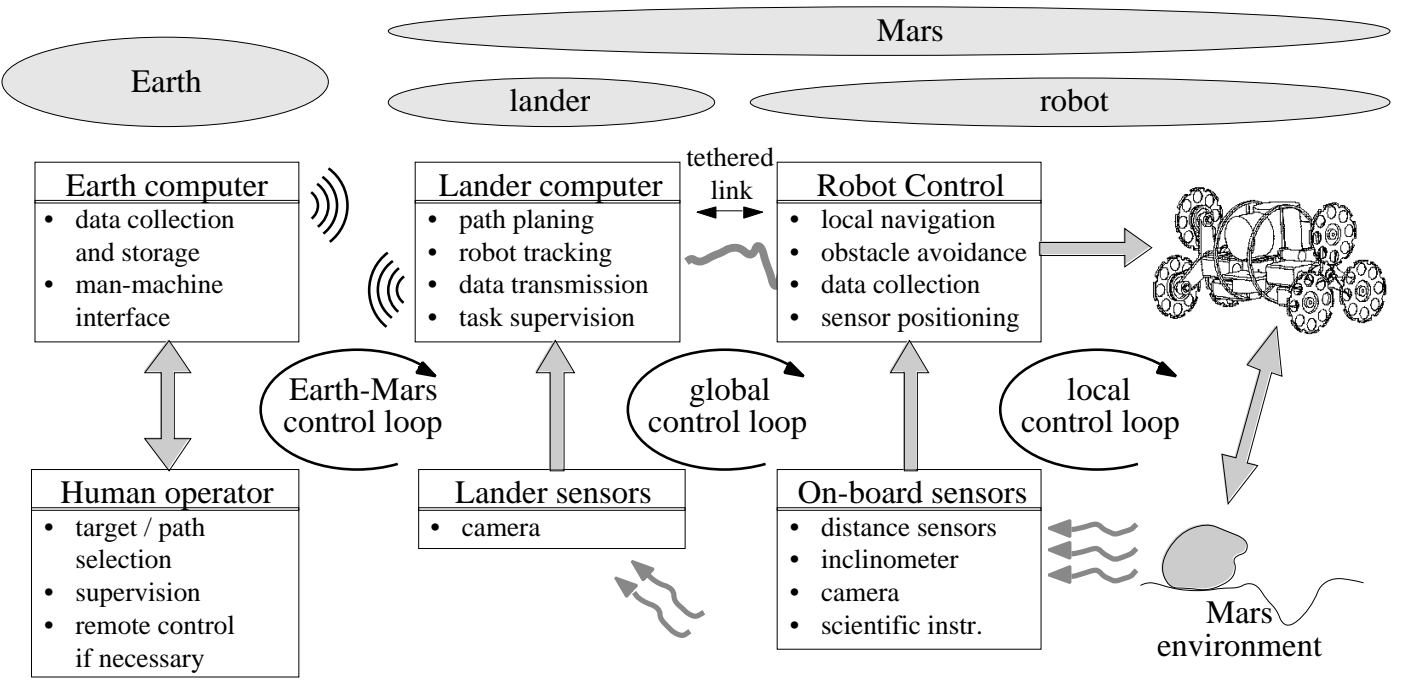

\section{FIGURE 6. Basic control concept of micro-rover}

The main task of the rover, which is to collect data with the on-board instruments, is defined as followed:

- Locations of scientific interest are determined by a teleoperator. Their position relative to the lander is known (assumed by the Earth planner). Therefore the difference of the rover's position and orientation with respect to the target is available for navigation (within the superposed tolerances).

- Path-planning is done by the teleoperator. The part to a certain location consists in a list of via points.

- The rover's position with respect to the lander is determinable by the lander with a precision of at least decimeters. Fine positioning, if necessary, is done with support of local (of rover on-board) sensor information.

To run the different tasks, the lander does the path processing, global navigation, collection and interpretation of vision data (lander camera) and position update (tracking of rover). The microrover controls the system within its local environment (local navigation), collects and interprets local sensor information, avoids obstacles, controls the motors and positions the scientific instruments. The link between lander and rover will mainly be used to submit all measurements taken with the on board scientific instruments to the lander and to update the rover's position measured with the camera on the lander.

A minimum of local intelligence has to be provided on the rover for reflexive reactions to critical situations like slipping downhill etc. Modules for turning, stepping over or on objects, recovery after flipping over and other maneuvers will be available. Simple obstacle avoidance algorithms based on data of simple and reliable local sensors are assumed to perform better than elaborated avoidance strategies with complex and delicate sensors. In that way it is possible to keep local control simple and reliable and the amount of data to be transferred to earth can be kept minimal.

\section{RESULTS AND CONCLUSIONS}

An innovative concept of a micro-rover for planetary exploration has been described. The introduction of stepping abilities to the wheeled robot allows for optimal locomotion on surfaces 
with little obstacles.

The breadboard model of the stepping triple wheels, its decentralized controller hardware and the man-machine interface have been implemented and are ready for testing.

\section{ACKLOWLEDGMENT}

This work was initiated and funded by the European Space Agency (ESA/ESTEC). It is accomplished by an interdisciplinary team consisting of von Hoerner \& Sulger in Schetzingen (prime contractor), the MPCh (Max-Planck-Institute for Chemistry) in Mainz, the DRL (German Aerospace Research Establishment) in Cologne and Wessling, MECANEX in Nyon and the Swiss Federal Institute of Technology in Lausanne. The authors like to thank all of them for their support and cooperation.

\section{REFERENCES}

[1] Matthies L., Gat E., Harrison R., "Mars Microrover Navigation: Performance Evaluation and Enhancement", in Proc. of the 1995 IEEE/RSJ International Conf. in Intelligent Robots and Systems, Vol. 1, 1995.

[2] Roll R., Van Winnendael M., Richter L., "The Development of an European Micro-Robot for Planetary Surface Exploration”, ASCE Robotics 98, Albuquerque, April 1998

[3] Chicarro A., Scoon G., Putz P.,"'Scientific Applications of Robotic Systems on Planetary Missions", Journal of Robotics and Automation Systems (Elsevier), Special Issue on Space Automation and Robotics, to be published early 1998

[4] Richter L. et. al.,'Mobile Micro-Rover for Scientific Instrument Deployment on Planets”, Journal of Robotics and Automation Systems (Elsevier), Special Issue on Space Automation and Robotics, to be published early 1998

[5] Schilling K., Jungius C., "Mobile Robots for Planetary Exploration", Control Engineering Practice, Vol. 4, No. 4, 1996.

[6] Burdick J.W., Radford J., and Chirikjian G.S., "A 'Sidewinding' Locomotion Gait for for HyperRedundant Robots", Proc. IEEE International Conference on Robotics and Automation, 1993.

[7] Desai R.S., Wilcox B., Bedard R., "JPL Robotic Vehicle Overview", in AUVS, 1992.

[8] McTamaney L.S., Douglas B.D., Harmon S.Y., "Mars Rover concept developement", Proc. SPIE Conf. 1007, Mobile Robots III, 1989.

[9] Spiessbach A., Clark B., Larimer S., Tobey B., Lindauer B., Koenig R., Lisec T., "Issues and Options for a Mars Rover", Proc. SPIE Conf. 852, Mobile Robots II, 1987.

[10] Wilcox B., Matthies L., Gennery D., Cooper B., Nguyen T., "Robotic Vehicles for Planetary Exploration", Proc. of the 1992 IEEE International Conf. on Robotics and Automation, 1992.

[11] Wright D.D., Watson R.E., "Comparison of Mobility System Concepts for a Mars Rover", Proc. SPIE Conf. 852, Mobile Robots II, 1987.

[12] Lee G.K., "System Requirements for Planetary Rovers", Proc. SPIE Conf. 1956, Sensor Fusion and Aerospace Applications, 1993.

[13] Nakayama R.,et. al.,’'Development of a Mobile Maintenance Robot System AIMARS, ASME, Proceeding of the USA-Japan Symposium on Flexible Automation, Minniapolis, 1988

[14] Meystel A. M., Isik C., "Pilot Level of a Hierarchical Controller for an Unmanned Mobile Robot", IEEE Journal of Robotics and Automation, Vol. 4, No. 3 June 1988

[15] Albus J.S., "RCS: A Reference Model Architecture for Intelligent Control", IEEE Journal of Computer Architectures for Intelligent Machines, May 1992

[16] Brooks R. A., "A Robust Layered Control System for a Mobile Robot", IEEE Journal of Robotics and Automation, Vol. RA.2, No. 1, March 1986

[17] Arkin R. C., "The Impact of Cybernetics on the Design of a Mobile Robot System: A Case Study", IEEE Transactions on Systems, Man, Cybernetics, Vol. 20, No. 6, November/December 1990

[18] Martin Alvarez A., de Peuter W., Putz P.,"A Unified Control Architecture for Planetary Rovers", 3rd International Symposium on Artificial Intelligence, Robotics and Automation for Space, iSAIRAS 94, Pasadena, October 1994 